\title{
Artists' Advance: Decreased Upper Alpha Power while Drawing in Artists Compared with Non-Artists
}

\author{
Mara Kottlow • Elke Praeg • Christine Luethy • \\ Lutz Jancke
}

Received: 10 January 2010/ Accepted: 1 October 2010/Published online: 14 October 2010

(C) Springer Science+Business Media, LLC 2010

\begin{abstract}
Brain mechanisms associated with artistic talents or skills are still not well understood. This exploratory study investigated differences in brain activity of artists and non-artists while drawing previously presented perspective line-drawings from memory and completing other drawing-related tasks. Electroencephalography (EEG) data were analyzed for power in the frequency domain by means of a Fast Fourier Transform (FFT). Low Resolution Brain Electromagnetic Tomography (LORETA) was applied to localize emerging significances. During drawing and related tasks, decreased power was seen in artists compared to non-artists mainly in upper alpha frequency ranges. Decreased alpha power is often associated with an increase in cognitive functioning and may reflect enhanced semantic memory performance and object recognition processes in artists. These assumptions are supported by the behavioral data assessed in this study and complement previous findings showing increased parietal activations in non-artists compared to artists while drawing. However, due to the exploratory nature of the analysis, additional confirmatory studies will be needed.
\end{abstract}

\footnotetext{
M. Kottlow ( $\square)$

Electrophysiology Section, Department of Psychiatric Neurophysiology, Psychiatric University Hospital Bern, Bolligenstrasse 111, 3000 Bern 60, Switzerland e-mail: kottlow@puk.unibe.ch

E. Praeg

Berenson-Allen Center for Noninvasive Brain Stimulation (CNBS), Beth Israel Deaconess Medical Center and Harvard Medical School, Boston, MA, USA

C. Luethy $\cdot$ L. Jancke

Department of Psychology, Neuropsychology, University

of Zurich, Zurich, Switzerland
}

Keywords Artists - Drawing - EEG $\cdot$ Alpha $\cdot$ Plasticity · LORETA

\section{Introduction}

Recently, there has been growing interest in collaboration between arts and sciences (Frazzetto and Anker 2009). Not only are artists getting inspired by scientific work, but scientists are also becoming interested in the secrets of artistic skills, represented for example by drawing.

Drawing a previously seen picture from memory combines different functions. Thus, along with visuo-motor processes, semantic and episodic memory as well as spatial attention processes may be required. In this article we want to concentrate on the former two processes, semantic memory and visuo-motor processes, which have been associated with oscillations in the upper alpha frequency range (Klimesch 1997; Klimesch 1999; Klimesch et al. 2005).

Drawing and motor imagery of drawing have been investigated with fMRI in untrained subjects. These studies revealed mainly activations of the parietal cortex and motor areas, while decreased or no activation was found in the temporal lobe (Ferber et al. 2007; Harrington et al. 2006; Makuuchi et al. 2003). Activations from the imagination of drawing were similar to that of real drawing tasks (Harrington et al. 2006). Also, during modeling, a process related to drawing, parietal areas have been shown to be more strongly activated than temporal regions in normal subjects (Jancke et al. 2001). Further, left frontotemporal lobar degeneration has been found to facilitate visual artistic skills in non-artists, indicating that the temporal lobe may not play an important role in the drawing process in non-artists or may inhibit artistic skills in untrained 
subjects (e.g. Gordon 2005; Mendez 2004; Miller et al. 2000). In contrast, previously trained artists showed no change of artistic skills after left frontotemporal degeneration (Finney and Heilman 2007).

Regarding artistic expertise, there are just a few studies directly comparing the brain activity of artists and nonartists (Bhattacharya and Petsche 2002; Bhattacharya and Petsche 2005; Bhattacharya 2009). Within these studies, EEG data was analyzed in terms of connectivity during mental imagination and visual perception. The drawing process itself, however, has not been compared between artists and non-artists.

In the present study, 16 artists and 16 non-artists were presented with different line-drawings which they had to draw from memory afterwards. They further completed drawing-related tasks such as visual imagination (VI) of the stimulus, motor imagination (MI) of the drawing process and non-object scribbling (adapted from Jancke et al. 2001). Relating our study to the EEG literature in the field, we roughly hypothesized effects to occur in the upper alpha frequency range during drawing and related tasks. The sources of upper alpha power were calculated applying LORETA. Thereby, particular attention was paid to the distribution of upper alpha power over the temporal and parietal visual association areas.

\section{Materials and Methods}

\section{Participants}

The experimental group consisted of 16 students or graduates of academies of fine arts ( 9 female), aged between 22 and 37 years (mean $28.5 \pm 3.83$ ). The control group comprised 16 students or graduates of higher education colleges or universities (9 female), aged between 22 and 39 years (mean $28.13 \pm 3.95$ ). All subjects were tested with the Annett (1970) and Bryden (1977) handedness questionnaires. Subjects were paid for participation. To guarantee their imaginativeness, they completed an imagination questionnaire for visual (Marks 1973) and motor imagination (designed by the investigators), which at the same time served as training for the later imagination task. Experiments were undertaken with the understanding and written consent of each subject and were approved by the local ethics committee.

\section{Stimuli and Tasks}

Prior to the experiment, 13 stimuli had been drawn and evaluated by the investigators. Drawings were controlled by a rating $(N=28)$ for similarity regarding the dimensions "familiarity", "complexity", "imagination coherency", and "emotional valuation" according to Gerlach et al. (2000). The subjects for the rating were not involved as subjects for the experiments. "Complexity" represented the rating of detailedness of the stimuli, "familiarity" meant how often the stimuli are encountered in reality or imagination, and "imagination coherency" intended the level of similarity between the mental picture of the stimulus and its illustration. The Kruskal-Wallis test of the ratings resulted in significant differences in all dimensions. We therefore chose nine stimuli from the original set, which resulted in similar values when applying the Kruskal-Wallis test for imagination coherency $(p=0.505)$ and valence $(p=0.162)$. Pictures differed in ratings of complexity $(p<0.1)$ and coherence $(p<0.05)$ and were therefore changed according to the raters' comments. The nine resulting similar stimuli are given in Fig. 1.

Because object recognition is faster if semantically similar objects are depicted (Boucart and Humphreys 1992; Pins et al. 2004), the stimuli were chosen to represent different kinds of rooms, as shown in Fig. 1. In order to facilitate object recognition, stimuli consisted of black and white line-drawings without shading, as according to Harley et al. (2004) it is easier to recognize stimuli with higher contrast. All pictures contained about nine objects to control for memory effects, one of them a real animate object (animal or plant).

The experiment was implemented using Presentation software (Neurobehavioral Systems, San Francisco, CA) and contained the conditions "drawing", "scribbling", visual imagination of the stimulus ("VI") and motor imagination of the drawing process ("MI"). The order of the conditions was pseudo-randomized among the subjects. During the condition "drawing" subjects were asked to copy the picture stimulus from their memory as accurately as possible, while during "scribbling" subjects just moved the pen inside the drawing area but were asked not to draw specific forms.

Subjects were seated in a height-adjustable chair with their chins supported by a chin rest in order to avoid head movements. For task performance, a computer keyboard and a $30 \times 30 \mathrm{~cm}$ drawing board with an angle of 25 degrees was placed in front of the subjects. On the drawing board 18 drawing sheets were placed. In the middle of each sheet a black frame of $7 \times 7 \mathrm{~cm}$ was printed, representing the drawing area for the conditions "scribbling" and "drawing". In the center of each square a fixation cross was printed to indicate the centre of fixation during rest. The drawing area was kept this small for reduction of eyemovement artifacts. However, eye movement artifacts while drawing were unavoidable, since visual feedback and the vision on one's own drawing movements influences the progression of movements and are thus important for 

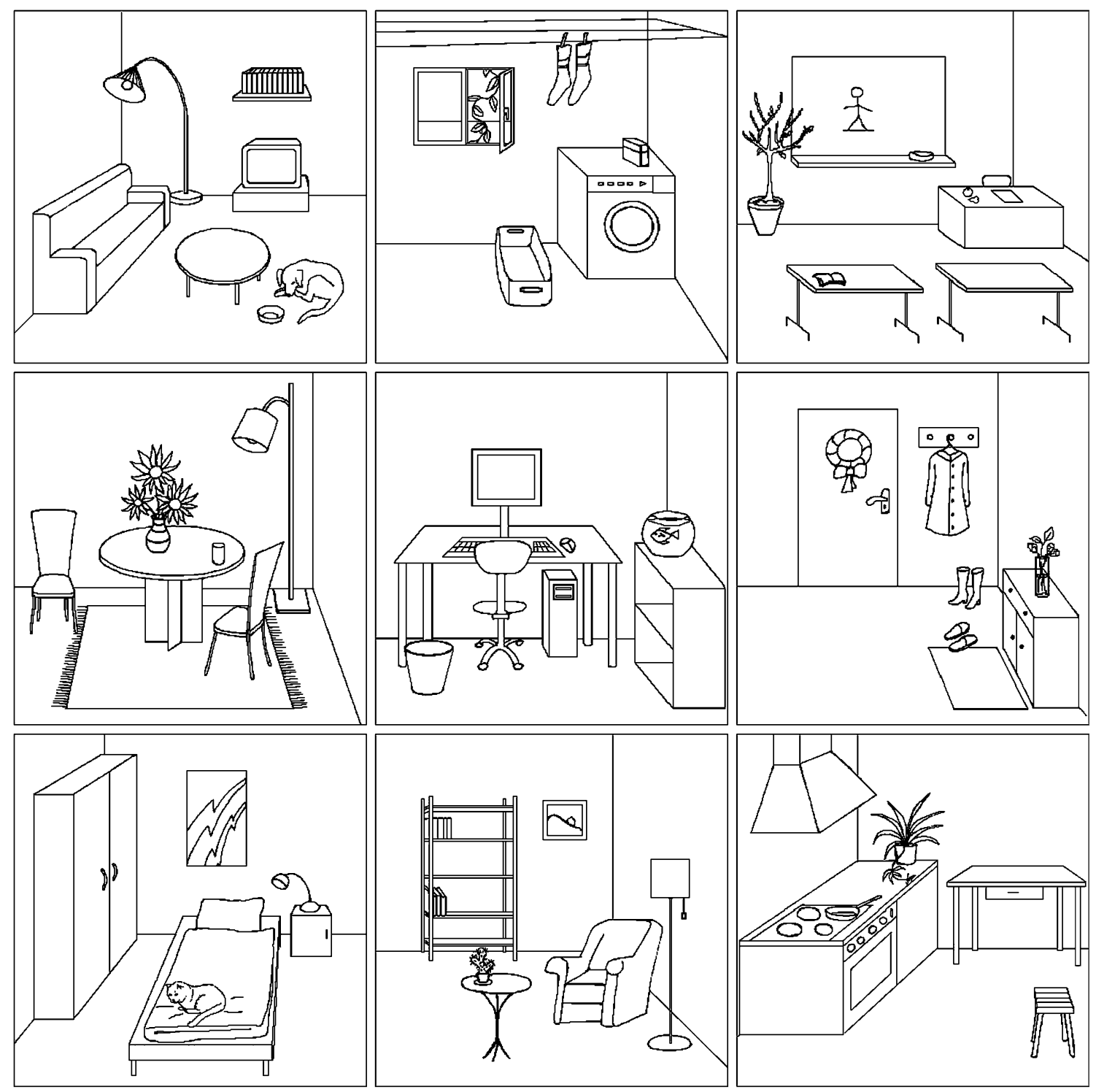

Fig. 1 The nine visual stimuli used for the experiment. Each picture represents a different kind of room. All pictures contained about nine objects to control for memory effects, among them one real animate object (animal or plant)

drawing (Smyth 1989). Subjects were given a black gel-ink roller for drawing, avoiding different nuances of darkness as often seen in pencil drawings and thus standardizing the drawing movements. The instructions for the motor imagination were adapted from Féry (2003).

The experiment took place in an illuminated room. Subjects were seated in front of a computer screen with their backs to the investigator, who noted the drawing order of the picture objects. In order to avoid auditory disturbances, subjects wore earplugs.

Before each condition written instructions presented on the computer screen informed the subjects about the following task. Subjects were able to choose the beginning of a condition by pressing a control button on the computer keyboard when ready to continue. Picture stimuli were presented for $30 \mathrm{~s}$ on a computer screen in the size $12.5 \times 12.5 \mathrm{~cm}$, corresponding to a visual angle of 7.85 degrees. Afterwards, a black background was presented on the screen that indicated the beginning of the active part of the trial (scribbling, drawing, visual/motor imagination). The duration of the conditions was $30,35,40$ or $45 \mathrm{~s}$ in pseudo-randomized order. The changing from black to white background indicated the end of the condition. The experiment consisted of nine different trials containing all five conditions. The order of the nine trials was randomized between subjects. The total duration of the experiment was about $45 \mathrm{~min}$. Figure 2 shows the experimental procedure on the example of one trial. 


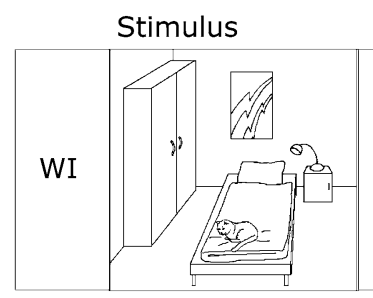

$30 \mathrm{~s}$

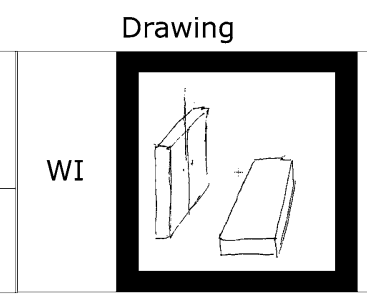

$30,35,40$ or $45 s$

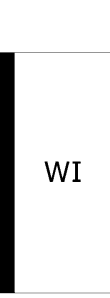

$30,35,40$ or $45 \mathrm{~s}$

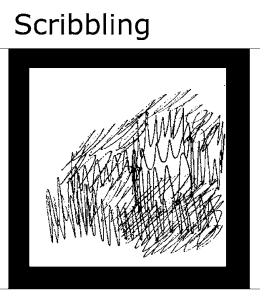

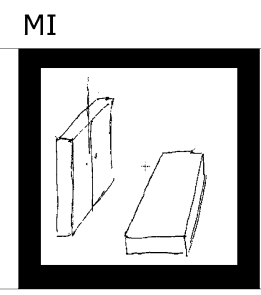

$30,35,40$ or $45 \mathrm{~s}$
VI

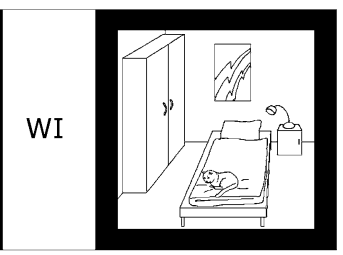

$30,35,40$ or $45 \mathrm{~s}$
Fig. 2 Task procedure. The picture shows the task procedure on the example of one trial. Each condition is always preceded by a written instruction (WI) appearing on the white computer screen. The tasks following the WI are started by the subject pressing a control button. The first condition is always represented by the stimulus presentation and lasts $30 \mathrm{~s}$. The following conditions are of a

\section{Behavioral Analyses}

Before the experiment, subjects had to complete a test for visual imagination (Marks 1973) and for motor imagination (designed by the authors). These tests consisted each of three scenarios that had to be imagined. Again three questions were asked for each scenario, which had to be answered on a five-point scale (one: very vivid imagination; five: no imagination). Tests scores were obtained calculating the mean rating for visual and motor imagination respectively. The imagination tests served additionally as practice for the later distinction between VI and MI tasks.

During the experiment, one of the investigators was seated behind the subject and listed which objects were drawn and in which order. This was the basis for the following scores:

(a) Number of objects drawn per session: total of objects divided into the categories "detail", "perspective", and "object in space" (objects contributing to the perspective)

(b) Drawing order ("started with detail" versus "started with perspective" or "started with object in space")

After the experiment, subjects were asked which and how many of the presented line-drawings they remembered and to list them in written form. Lastly, they had to rate the previously presented line-drawings on the dimensions complexity, familiarity and emotional valuation as described in the methods section "stimuli and task" (the dimension "imagination coherence" has been removed from the rating because it seemed difficult to understand).

\section{EEG Data Acquisition and Preprocessing}

EEG was recorded using 30 scalp electrodes arranged according to a subset of the 10-10 system, which were attached to an electrode cap (Easy Cap System, Falk random duration $(30,35,40$ or $45 \mathrm{~s})$. Also the order of these conditions is random. The duration of the conditions drawing, scribbling, MI and VI was indicated through the darkening and enlightening of the screen, as illustrated by the black borders on the scheme. WI Written instructions, $s$ seconds, MI motor imagination, $V I$ visual imagination

Minow Services, FMS). The 32-channel amplifier from Brain Products GmbH (Brain Vision LLC, Virginia) was accessed by a recording computer. To measure eye movements, two ocular electrodes were attached bilaterally below both canthi. For recording and analysis Brain Vision Recorder and Analyzer software were used (electrode impedance $<5 \mathrm{k} \Omega$, low and high pass filter $0.1-70 \mathrm{~Hz}$, recording frequency $500 \mathrm{~Hz}$ ). The raw data was filtered offline with a frequency range of $0.5-30 \mathrm{~Hz}$ including a $50 \mathrm{~Hz}$ Notch filter. The original reference was FCz. To clean the data of eye movement artifacts, an ICA (Independent Component Analysis) was employed. Remaining artifacts were manually removed. The EEG data of all but the ocular electrodes were transformed to the average reference. For each subject, EEG data was analyzed separately for each condition. In a first step, each of the nine trials of each condition was divided into segments of 1024 data points.

\section{EEG and LORETA Analyses}

Frequency domain EEG data was transformed by means of a Fast Fourier Transform (FFT; Brain Vision Analyzer, Brain Products GmbH, Gilching) applied on the segments of 1024 data points to reveal the power spectra $\left(\mu V^{2}\right)$ from 1 to $30 \mathrm{~Hz}$. FFT was computed for non complex data using the full spectrum and the maximal resolution of $0.488 \mathrm{~Hz}$ with a $10 \%$ window length (Hanning window). The segments of each subject and condition were averaged and analyzed computing a TANOVA (topographic analysis of variance; described in Stein et al. 2006) for the factors "condition" (drawing, picture, scribbling, VI, MI) and "group" (artists and non-artists) over log transformed spectral amplitudes averaged in the following frequency ROIs defined according to the default LORETA frequency settings (delta: $1.5-6 \mathrm{~Hz}$; theta: $6.5-8.0 \mathrm{~Hz}$; lower alpha: 8.5-10 Hz; upper alpha: $10.5-12 \mathrm{~Hz}$; beta 1: $12.5-18 \mathrm{~Hz}$; beta 2 : $18.5-21 \mathrm{~Hz}$; beta $3: 21.5-30 \mathrm{~Hz}$ ). The TANOVA 
computes the differences of EEG topographies (Lehmann 1987; Murray et al. 2008) and defines its significances with a nonparametric randomization test correcting for multiple comparisons (Manly 1997). Frequency bands showing significant results on the factor "group" were compared between artists and non-artists by means of a $t$-test in each condition.

LORETA (Pascual-Marqui et al. 1994) was used to estimate the sources of the significant differences found by means of the TANOVA. LORETA has been validated by studies comparing the results with results from fMRI (e.g. Mulert et al. 2005), structural MRI (Worrell et al. 2000) and positron emission tomography (PET) (Zumsteg et al. 2005). It computes electrical activity by assuming similar activation among neighboring neuronal clusters. A threeshell spherical head model and EEG electrode coordinates derived from cross-registrations between spherical and realistic head geometry were utilized, which were both registered to the digitized Montreal Neurological Institute (MNI) standard brain. LORETA also computed an exceedance proportion test (Friston et al. 1991) with correction for multiple testing using randomization of the maximum-statistic. This test reported a collection of threshold values and the corresponding probabilities that the number of suprathreshold voxels is significant.

\section{Results}

\section{Behavioral Data}

Artists $(N=16$; mean $(M)=1.9 \pm 0.4)$ scored by trend better than non-artists $(N=16 ; M=2.2 \pm 0.4)$ in visual imagination $(p<0.1)$. No differences were found in motor imagination (artists $M=2.0 \pm 0.7$; non-artists $M=$ $2.0 \pm 0.5)$.

Regarding the total number of drawn objects, artists $(N=16 ; M=5.64 \pm 1.24)$ performed significantly better than non-artists $(N=15$, one subject excluded due to missing recordings of drawing details; $M=4.62 \pm 1.20$; $p<0.05)$. Unpaired $t$-tests were conducted for each category (perspective, object in space, detail) comparing both groups. The results revealed that artists drew significantly more details than non-artists $(p<0.01$; artists 34\%, nonartists $27 \%$ ). Non-artists in contrast to artists orientated themselves on the perspective of the stimuli, which consisted of the lines building the room or perspective furniture (artists $24 \%$, non-artists 30\%).

The evaluation of the drawing order of objects contained also the same three object categories: perspective, object in space and detail. We compared the number of drawings initiated with perspective, object in space or detail between artists and non-artists by means of a Mann-Whitney $U$-test.
It turned out that artists compared with non-artists tended to begin their drawings more often with a detail $(p<0.1$; artists $8 \%$, non-artists $2 \%$ ). Non-artists tended to begin their drawings more frequently with perspective lines ( $p<0.1$; artists $60 \%$, non-artists 67\%). Examples of an artist's and a non-artist's drawing and scribbling, respectively, are illustrated in Fig. 3.

After the experiment we tested the memory for the presented stimuli. We found no difference between groups (artists $M=6.3 \pm 1.3$; non-artists $M=6.1 \pm 1.5$ ).

The rating of the visual stimuli by the participants was analyzed by means of the Kruskal-Wallis-test. It showed significant differences concerning familiarity and emotional valuation $(p<0.05)$, but similar ratings in complexity $(p=0.193)$ for all subjects rating the stimuli $(N=30$; one subject of each group excluded due to missing values). Analyzed within each group, the ratings resulted in very similar valuation of complexity $(p=0.367)$ and emotional valuation $(p=0.262)$ and only weak differences concerning familiarity $(p=0.089)$ in the group of non-artists $(N=15)$. The Kruskal-Wallis-test for the group of artists $(N=15)$ showed significant differences in the dimensions familiarity $(p<0.05)$ and emotional valuation $(p<0.001)$. Concerning complexity the stimuli have been regarded as homogeneous $(p=0.322)$. The average ratings of artists and non-artists have been compared by $t$-tests for each rating-item and did not differ between groups. The exact $\mathrm{p}$ values were $p=0.508$ for complexity, $p=0.465$ for familiarity, and $p=0.606$ for emotional evaluation.

\section{Spectral EEG Analyses}

The TANOVA showed a global effect for the factor "group" in the upper alpha frequency band from 10.5 to $12 \mathrm{~Hz}(p<0.05)$. The other frequency ROIs did not show any significant "group" main effects (delta: $p=0.935$; theta: $p=0.217$; lower alpha: $p=0.704$; beta $1: p=$ 0.108 ; beta $2: p=0.841$; beta $3: p=0.317$ ). Driven by these effects we conducted $t$-tests for each condition and frequency step between artists and non-artists over the frequencies from 10.7 to $12.3 \mathrm{~Hz}$ (the difference in the frequency range compared to the other analyses is due to zero padding).

As shown in Fig. $4 \mathrm{c}$, the $t$-test of the power maps from 10.7 to $12.3 \mathrm{~Hz}$ of artists and non-artists during drawing showed a significant decrease of power in artists $(t(15)>2.13$ for $p<0.05)$. This effect was seen at right occipitoparietal $(10.7-11.7 \mathrm{~Hz})$ and central frontal electrodes (11.7-12 Hz). Accordingly, similar results were obtained for the conditions VI and MI, but not for scribbling and picture presentation, where no differences in this frequency range were found. 
Fig. 3 Representative selection of artist's (a) and non-artists's (b) scribblings and drawings illustrating the difference in their drawing skills
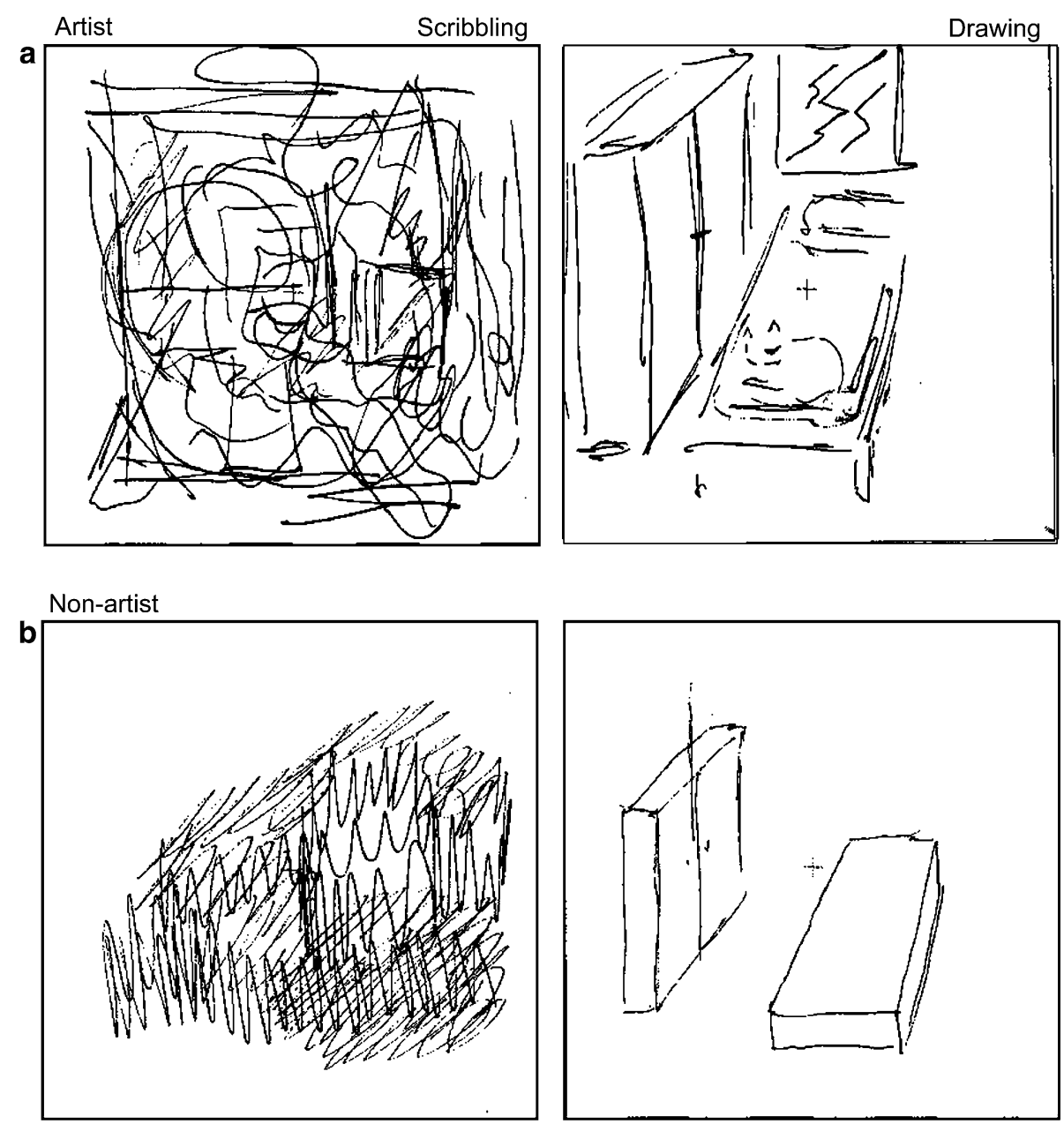

Figure 4 shows the spectral power maps (10.7-12.3 Hz) for artists (a) and non-artists (b). To illustrate the significance of these findings, a time-frequency analyses was conducted over all frequencies comparing artists and nonartists. The time-frequency plot is shown in Fig. 5 and serves only the purpose of illustration of the frequencies' behaviour; time-based effects cannot be applied on the drawing functions as analyzed in this study.

\section{LORETA}

Since the TANOVA resulted in a significant main effect on the factor "group" in the upper alpha band (10.5-12 Hz), we computed LORETA analyses for this frequency band for all conditions to roughly localize the sources.

A significant difference in upper alpha power was seen during drawing in inferior and middle temporal areas of the right hemisphere, consisting of decreased upper alpha power in artists compared to non-artists. The localized regions and the corresponding $t$-values are listed in Table 1.
During VI and MI, decreased upper alpha power was revealed in artists mainly in right hemispheric primary and secondary visual areas, in right inferior and left middle temporal regions and in the right posterior cingulate cortex. During picture presentation and scribbling no significant differences in upper alpha power were revealed using LORETA.

The exceedence proportion test computed in LORETA resulted in $p$ (cls) $<0.05$ for upper alpha effects of the contrast artists versus non-artists for drawing, MI and VI.

\section{Discussion}

Within this EEG study we analyzed the differences in brain activity of artists and non-artists while drawing and performing drawing-related tasks. The analysis has been based on the a priori hypothesis that the differences would mainly be observed in the upper alpha band, which is supported by the data. However, other hypotheses, affecting other frequency bands are conceivable, such that problems of 
Fig. 4 Spectral power scalp maps $\left(\mu \mathrm{V}^{2}\right)$ from 10.7 to $12.2 \mathrm{~Hz}$ of artists (a) and nonartists (b) during drawing. Red indicates positive power values (scale below each image).

c T-maps of the contrast artists versus non-artists during drawing $(t(15)>2.13$ for $p<0.05$ ), $t$-value scale below the image. Blue: significant power decrease in artists, red: significant power increase in artists a
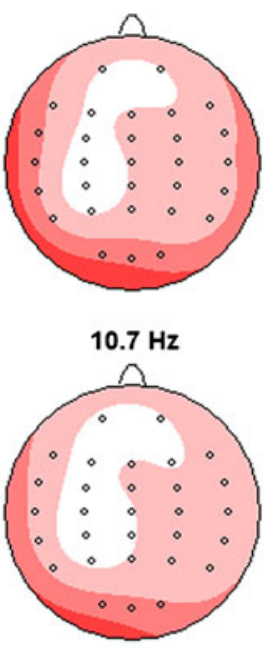

11.2 Hz

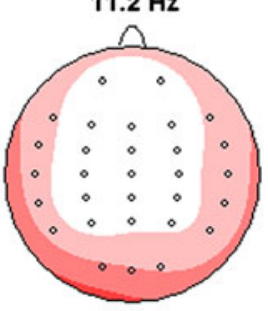

$11.7 \mathrm{~Hz}$

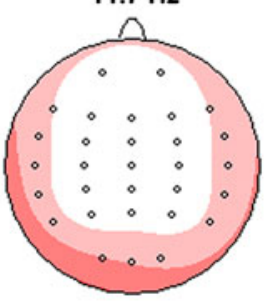

$12.2 \mathrm{~Hz}$ b

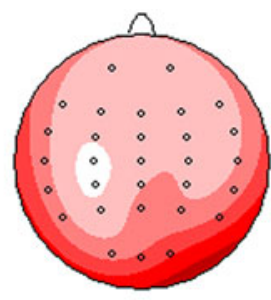

10.7 Hz

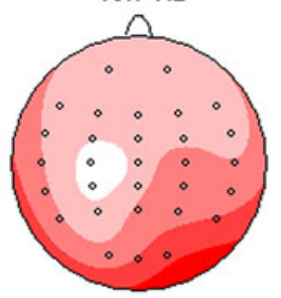

11.2 Hz

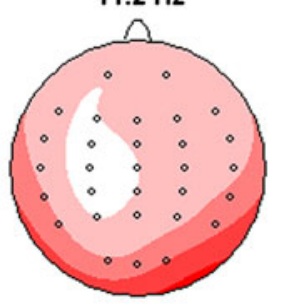

$11.7 \mathrm{~Hz}$

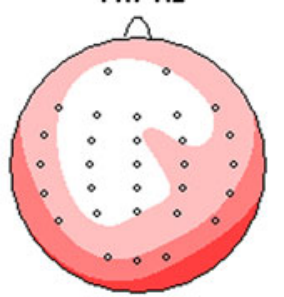

$12.2 \mathrm{~Hz}$
C

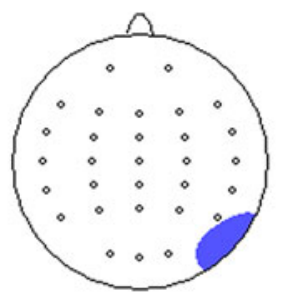

$10.7 \mathrm{~Hz}$

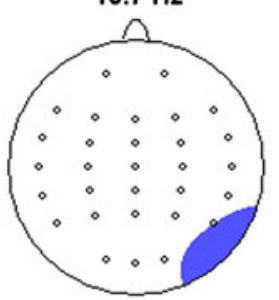

11.2 Hz

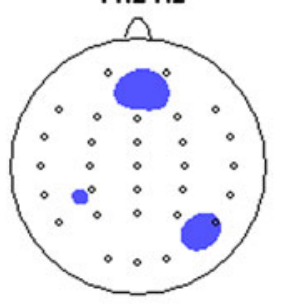

$11.7 \mathrm{~Hz}$

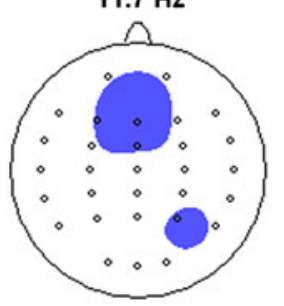

$12.2 \mathrm{~Hz}$

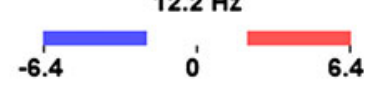

6.4

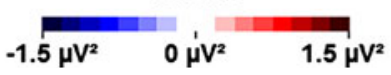

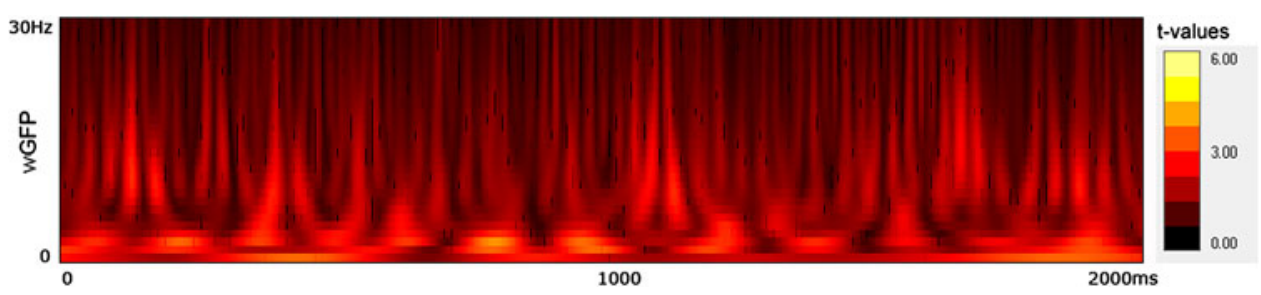

Fig. 5 Time-frequency plot of the spectral activity from 0 to $30 \mathrm{~Hz}$ of drawing in artists versus non-artists $(t(15)>2.13$ for $p<0.05)$. The picture shows the average $2 \mathrm{~s}$ of the drawing process (the total

multiple testing across the seven frequency bands may have occurred. For a more conservative discussion of the issues raised here, further confirmatory studies are necessary.

The mean number of drawn objects is an indicator for the pace of drawing as well as for semantic memory duration of the drawing conditions was between 30 and $45 \mathrm{~s}$ ). The color bar on the right shows the colors for the respective $t$-values. $w G F P$ global field power represented by wavelets

capacity. As seen in the drawings of artists and non-artists, artists drew significantly more details. This may be explained by a better semantic memory, although drawing speed may be generally enhanced in artists compared to non-artists. With these behavioral results we support the findings of a visual memory study from Vogt and 
Table 1 Localization of upper alpha power differences between groups during drawing regions of significantly decreased upper alpha power $(t(15)>2.13$ for $p<0.05)$ in artists compared with non-artists during the condition drawing localized by LORETA

\begin{tabular}{|c|c|c|c|c|c|}
\hline \multirow[t]{2}{*}{ Anatomical area } & \multirow[t]{2}{*}{ BA } & \multicolumn{3}{|c|}{ Talairach coordinates } & \multirow[t]{2}{*}{$t$-value } \\
\hline & & $\mathrm{x}$ & $\mathrm{y}$ & $\mathrm{z}$ & \\
\hline \multicolumn{6}{|l|}{ Upper alpha power } \\
\hline Middle temporal gyrus & 21 & 60 & -60 & 8 & -2.81 \\
\hline Middle temporal gyrus & 21 & 60 & -60 & 1 & -2.79 \\
\hline Middle temporal gyrus & 39 & 53 & -60 & 8 & -2.81 \\
\hline Inferior temporal gyrus & 37 & 60 & -60 & -13 & -2.81 \\
\hline
\end{tabular}

$B A$ Brodman's areas

Magnussen (2007) showing that there is no overall difference in the number of remembered pictures, but in the number of correctly recalled pictorial features.

According to the analysis of the subjects' stimulus rating, the visual stimuli can be regarded as standardized for non-artists in all three dimensions (complexity, familiarity and emotional valuation). In contrast, artists seem to apply different criteria as ratings varied between the different pictures regarding familiarity and emotional valuation. This may be due to their training in processing pictorial material and could be the subject of further studies.

As hypothesized, the upper alpha frequency band power differed significantly between artists and non-artists. Upper alpha power was significantly decreased in artists during drawing, MI, and VI; with similar topographies during all conditions. The sources of the upper alpha effect, according to LORETA, lay within the right inferior and middle temporal gyrus for drawing, within the right inferior and left middle temporal gyrus for VI, and within the right inferior temporal gyrus for MI. For MI and VI sources have further been found in primary and secondary visual areas and in the right posterior cingulate gyrus. The results thus show a similarity between task performance and imagination conditions, which has also been found by Jancke et al. (2001) and Bhattacharya (2009). VI and MI induced almost the same theta and upper alpha effects. Subjects may have had difficulties in the distinction of these two tasks and may therefore have imagined visual as well as motor processes in both tasks.

Alpha power has been suggested to inversely correlate with cortical activity as measured with fMRI (Laufs et al. 2003). This is particularly the case for regions of the socalled default mode network which involve the anterior and posterior cingulate cortex, orbitofrontal and parietotemporal areas, the superior frontal gyrus, the insula, the supramarginal gyrus and the supplementary motor areas (Jann et al. 2009). Within these regions, alpha is suppressed by eye opening, visual stimuli and visual scanning, whereas it is known to be enhanced during internal tasks, such as mental calculation and working memory. Oscillations in the alpha frequency range have thus been thought to reflect inhibition of task-irrelevant cortical areas (Palva and Palva 2007). Alpha levels at rest and its consequent response during tasks vary in different subjects. During EEG at rest, higher alpha power has been associated to high cognitive capacity, whereas during cognitive tasks a decrease in alpha power was present in good performers (Klimesch 1999). Alpha has further been related to spatial attention (Rihs et al. 2007), semantic memory (Klimesch et al. 1993; Klimesch et al. 1997) and visual discrimination performance (Hanslmayr et al. 2005). Thus, different aspects of spatial attention can either cause decreases or increases of alpha power over occipito-parietal electrodes (Rihs et al. 2007). Further, decreases in upper alpha power compared to baseline are associated with enhanced semantic memory functions in one study (Klimesch et al. 1997), while in an other study, this effect seems to correlate specifically with bad performance (Klimesch et al. 1993). A decrease in alpha power has also been associated to better visual discrimination performance (Hanslmayr et al. 2005). Holding these varying results in mind, the decrease in upper alpha power during drawing may possibly reflect an enhanced recall from semantic memory of previously stored shapes in artists compared to non-artists obtained by extensive practice. This is supported by the localization of the alpha effects to the temporal lobe in all three conditions, since the right inferior temporal gyrus has been found to be responsible for feature and object discrimination and for the integration of visuospatial perception (Mendez 2004). The behavioral results showing more drawn objects and pictorial details by artists support this conclusion. Further, the alpha decrease in left middle temporal areas in artists during VI and MI may reflect a better performance of imagination tasks in artists compared with non-artists. This is by trend supported by the behavioural VI scores.

The spatial distribution of the effects found in this study can be addressed in light of the neural efficiency hypothesis (Haier et al. 1988) which originally explained brain plasticity in the context of training. There is a whole branch of research on practice and expertise as well as on intelligence which may be comparable to talent. Recently, Neubauer and Fink (2009) and Kelly and Garavan (2005) concluded in their reviews that there is no clear evidence for a pattern of decrease or increase of brain activation related to practice, intelligence or expertise. Various studies reported brain activation decrease with practice or in brighter subjects (Grabner et al. 2004; Lengger et al. 2007), while other studies reported brain activation increases in task-relevant brain areas (Debaere et al. 2004) or activation decreases in some brain regions and increases in others (Poldrack and Gabrieli 2001). Moderating variables of these changes may 
be sex, task type, task complexity, task difficulty or brain area. Neural efficiency arises in particular when subjects are confronted with subjectively low to moderate task difficulty. In complex tasks more able individuals seem to invest more cortical resources resulting in positive correlations between brain usage and cognitive ability (Doppelmayr et al. 2000; Doppelmayr et al. 2005; Gray et al. 2003; Larson et al. 1995; Lee et al. 2006; Neubauer et al. 1999). Similarly, Lamm et al. (2001) claim that the neural efficiency hypothesis may only be supported in timeunrestricted conditions. In restricted conditions, good performers may invest additional effort and consequently gain more correct responses on a behavioural level. Thereby, the investment of cortical effort may be a volitional decision of the individual. Practice of motor tasks may result in low recruitment of frontal executive functions when the task processing occurs in an automated manner (Kelly and Garavan 2005; Ross et al. 2003). This is however not the case in our study since the movements for drawing are variable and may never become automated.

Effects revealed by our study are found mainly on the right hemispheres of topographies and LORETA images. This is in-line with findings from Harrington et al. (2006) and from lesion studies on artists suffering from righthemispheric distortions (e.g. Berti et al. 2007; KleinerFisman et al. 2003; Kleiner-Fisman and Lang 2004). Moreover, most effects were localized within temporal areas. As mentioned before, drawing and related tasks have been found to activate parietal regions omitting the temporal lobe in untrained subjects (Ferber et al. 2007; Harrington et al. 2006; Makuuchi et al. 2003). Since no differences were located within parietal regions, we conclude that in both artists and non-artists the parietal lobe may equally contribute to drawing processes and related tasks. The differences localized in the temporal lobe may indicate that this region is possibly involved in such tasks in artists only. It may further be an indicator for a higher amount of interaction between ventral and dorsal visual streams in artists. This assumption is based on a recent study from Himmelbach and Karnath (2005), which has shown a contribution of the ventral visual stream to visuomotor processing and target-oriented movements under certain conditions.

\section{Conclusion}

Within this study, the actual drawing process has been compared between artists and non-artists for the first time. These exploratory results show significant differences in the amount and distribution of upper alpha power during drawing, MI and VI, but not during the control conditions of scribbling and picture viewing. This pattern suggests a differently distributed network for drawing in artists as compared to non-artists.

The processes examined in our study are very complex and require inseparable motor and cognitive functions, which cannot be clearly divided. Nevertheless, this study reveals interesting neuronal and functional differences in active and mental drawing tasks between artists and nonartists.

Acknowledgements This work was financed by the Swiss National Science Foundation (Project "Short-term and long-term plasticity in the sensorimotor cortex") as part of the NCCR (National Center of Competence in Research "Neural plasticity and repair") grant.

\section{References}

Annett M (1970) A classification of hand preference by association analysis. Br J Psychol 61:303-321

Berti A, Cappa SF, Folegatti A (2007) Spatial representations, distortions and alterations in the graphic and artistic production of brain-damaged patients and of famous artists. Funct Neurol $22: 243-256$

Bhattacharya J (2009) Increase of universality in human brain during mental imagery from visual perception. PLoS One. doi:10.1371/ journal.pone.0004121

Bhattacharya J, Petsche H (2002) Shadows of artistry: cortical synchrony during perception and imagery of visual art. Cogn Brain Res 13:179-186

Bhattacharya J, Petsche H (2005) Drawing on mind's canvas: differences in cortical integration patterns between artists and non-artists. Hum Brain Mapp 26:1-14

Boucart M, Humphreys GW (1992) Global shape cannot be attended without object identification. J Exp Psychol Hum Percept Perform 18:785-806

Bryden MP (1977) Measuring handedness with questionnaires. Neuropsychologia 15:617-624

Debaere F, Wenderoth N, Sunaert S, Van Hecke P, Swinnen SP (2004) Changes in brain activation during the acquisition of a new bimanual coodination task. Neuropsychologia 42:855-867

Doppelmayr M, Klimesch W, Schwaiger J, Stadler W, Röhm D (2000) The time locked theta response reflects interindividual differences in human memory performance. Neurosci Lett 278:141-144

Doppelmayr M, Klimesch W, Sauseng P, Hödlmoser K, Stadler W, Hanslmayr S (2005) Intelligence related differences in EEGbandpower. Neurosci Lett 381:309-313

Ferber S, Mraz R, Baker N, Graham SJ (2007) Shared and differential neural substrates of copying versus drawing: a functional magnetic resonance imaging study. Neuroreport 18:1089-1093

Féry Y-A (2003) Differentiating visual and kinesthetic imagery in mental practice. Consciousness Exper Psychol 57:1-10

Finney GR, Heilman KM (2007) Artwork before and after onset of progressive nonfluent Aphasia. Cogn Behav Neurol 20:7-10

Frazzetto G, Anker S (2009) Neuroculture. Nat Rev Neurosci 10:815-821

Friston KJ, Frith CD, Liddle PF, Frackowiak RSJ (1991) Comparing functional (PET) images: the assessment of significant change. J Cereb Blood Flow Metab 11:690-699

Gerlach C, Law I, Gade A, Paulson OB (2000) Categorization and category effects in normal object recognition: a PET study. Neuropsychologia 38:1693-1703 
Gordon N (2005) Unexpected development of artistic talents. Postgrad Med J 81:753-755

Grabner RH, Fink A, Stipacek A, Neuper C, Neubauer AC (2004) Intelligence and working memory systems: evidence of neural efficiency in alpha band ERD. Cogn Brain Res 20:212-225

Gray JR, Chabris CF, Braver TS (2003) Neural mechanisms of general fluid intelligence. Nat Neurosci 6:316-322

Haier RJ, Siegel BV, Nuechterlein KH, Hazlett E, Wu JC, Paek J, Browning HL, Buchsbaum MS (1988) Cortical glucose metabolic rate correlates of abstract reasoning and attention studied with positron emission tomography. Intelligence 12:199-217

Hanslmayr S, Klimesch W, Sauseng P, Gruber W, Doppelmayr M, Freunberger R, Pecherstorfer T (2005) Visual discrimination performance is related to decreased alpha amplitude but increased phase locking. Neurosci Lett 375:64-68

Harley EM, Dillon AM, Loftus GR (2004) Why is it difficult to see in the fog? How stimulus contrast affects visual perception and visual memory. Psychon Bull Rev 11:197-231

Harrington GS, Farias D, Davis CH, Buonocore MH (2006) Comparison of the neural basis for imagined writing and drawing. Hum Brain Mapp 28:450-459

Himmelbach M, Karnath HO (2005) Dorsal and ventral stream interaction: contributions from optic ataxia. J Cogn Neurosci 17:632-640

Jancke L, Kleinschmidt A, Mirzazade S, Sha NJ, Freund H-J (2001) The role of the inferior parietal cortex in linking the tactile perception and manual construction of object shapes. Cereb Cortex 11:114-121

Jann K, Dierks T, Boesch C, Kottlow M, Strik W, Koenig T (2009) BOLD correlates of EEG alpha phase-locking and the fMRI default mode network. NeuroImage 45:903-916

Kelly AM, Garavan H (2005) Human functional neuroimaging of brain changes associated with practice. Cereb Cortex 15: 1089-1102

Kleiner-Fisman G, Lang AE (2004) Insights into brain function through the examination of art: the influence of neurodegenerative diseases. Neuroreport 15:933-937

Kleiner-Fisman G, Black SE, Lang AE (2003) Neurodegenerative disease and the evolution of art: the effects of presumed corticobasal degeneration in a professional artist. Mov Disord 18:294-302

Klimesch W (1997) EEG-alpha rhythms and memory processes. Int J Psychophysiol 26:319-340

Klimesch W (1999) EEG alpha and theta oscillations reflect cognitive and memory performance: a review and analysis. Brain Res Rev 29:169-195

Klimesch W, Schimke H, Pfurtscheller G (1993) Alpha frequency, cognitive load and memory performance. Brain Topogr 5:241-251

Klimesch W, Doppelmayr M, Pachinger T, Russegger H (1997) Event-related desynchronization in the alpha band and the processing of semantic information. Cogn Brain Res 6:83-94

Klimesch W, Hanslmayr S, Sauseng P, Gruber W, Brozinsky CJ, Kroll NEA, Yonelinas AP, Doppelmayr M (2005) Oscillatory EEG correlates of episodic trace decay. Cereb Cortex 16: 280-290

Lamm C, Bauer H, Vitouch O, Durec S, Gronister R, Gstättner R (2001) Restriction of task processing time affects cortical activity during processing of a cognitive task: an event-related slow cortical potential study. Cogn Brain Res 10:275-282

Larson GE, Haier RJ, Lacasse L, Hazen K (1995) Evaluation of a "Mental Effort" hypothesis for correlations between cortical metabolism and intelligence. Intelligence 21:267-278

Laufs H, Kleinschmidt A, Beyerle A, Eger E, Salek-Haddadi A, Preibisch C, Krakow K (2003) EEG-correlated fMRI of human alpha activity. NeuroImage 19:1463-1476
Lee KH, Choi YY, Gray JR, Cho SH, Chae JH, Lee S, Kim K (2006) Neural correlates of superior intelligence: stronger recruitment of posterior parietal cortex. NeuroImage 29:578-586

Lehmann D (1987) Principles of spatial analysis. In: Gevins AS, Remond A (eds) Handbook of electroencephalography and clinical neurophysiology, vol. 1: methods of analysis of brain electrical and magnetic signals. Elsevier, Amsterdam, pp 309-354

Lengger PG, FPhS Fischmeister, Leder H, Bauer H (2007) Functional neuroanatomy of the perception of modern art: a DC-EEG-study on the influence of stylistic information on aesthetic experience. Brain Res 1158:93-102

Makuuchi M, Kaminaga T, Sugishita M (2003) Both parietal lobes are involved in drawing: a functional MRI study and implications for constructional apraxia. Cogn Brain Res 16:338-347

Manly BFJ (1997) Randomization. Bootstrap and Monte Carlo Methods in Biology, Chapman and Hall, London

Marks DF (1973) Visual imagery differences in the recall of pictures. Br J Psychol 64:17-24

Mendez MF (2004) Dementia as a window to the neurology of art. Med Hypotheses 63:1-7

Miller BL, Boone K, Cummings JL, Read SL, Mishkin F (2000) Functional correlates of musical and visual ability in frontotemporal dementia. Br J Psychiatry 176:458-463

Mulert C, Menzinger E, Leicht G, Pogarell O, Hegerl U (2005) Evidence of a close relationship between conscious effort and anterior cingulate cortex activity. Int J Psychophysiol 56:65-80

Murray CC, Brunet D, Michel CM (2008) Topographic ERP Analyses: a step-by-step tutorial review. Brain Topogr 20: 249-264

Neubauer AC, Fink A (2009) Intelligence and neural efficiency. Neurosci Biobehav Rev 33:1004-1023

Neubauer AC, Sange G, Pfurtscheller G (1999) Psychometric intelligence and event-related desynchronization during performance of a letter matching task. In: Pfurtscheller G, Lopes da Silve FH (eds) Event-related desynchronization (ERD) and related oscillatory EEG-phenomena of the awake brain. Elsevier, Amsterdam, pp 219-231

Palva S, Palva JM (2007) New vistas for alpha-frequency band oscillations. Trends Neurosci 30:150-158

Pascual-Marqui RD, Michel CM, Lehmann D (1994) Low resolution electromagnetic tomography: a new method for localizing electrical activity in the brain. Int J Psychophysiol 18:49-65

Pins D, Meyer ME, Foucher J, Humphreys G, Boucart M (2004) Neural correlates of implicit object identification. Neuropsychologia 42:1247-1259

Poldrack RA, Gabrieli JD (2001) Characterizing the neural mechanisms of skill learning and repetition priming: evidence from mirror reading. Brain 124:67-82

Rihs TA, Michel CM, Thut G (2007) Mechanisms of selective inhibition in visual spatial attention are indexed by alpha-band EEG synchronization. Eur J Neurosci 25:603-610

Ross JS, Tkach J, Ruggieri PM, Lieber M, Lapresto E (2003) The mind's eye: functional MR imaging evaluation of golf motor imagery. Am J Neuroradiol 24:1036-1044

Smyth MM (1989) Visual control of movement patterns and the grammar of action. Acta Psychol 70:253-265

Stein M, Dierks T, Brandeis D, Wirth M, Strik W, Koenig T (2006) Plasticity in the adult language system: a longitudinal electrophysiological study on second language learning. NeuroImage 33:774-783

Vogt S, Magnussen S (2007) Expertise in pictorial perception: eyemovement patterns and visual memory in artists and laymen. Perception 36:91-100

Worrell GA, Lagerlund TD, Sharbrough FW, Brinkmann BH, Busacker NE, Cicora KM, O'Brien TJ (2000) Localization of 
the epileptic focus by low-resolution electromagnetic tomography in patients with a lesion demonstrated by MRI. Brain Topogr $12: 273-282$
Zumsteg D, Wennberg RA, Buck A, Wieser HG (2005) $\mathrm{H}_{2}(15) \mathrm{O}$ or 13NH3 PET and electromagnetic tomography (LORETA) during partial status epilepticus. Neurology 65:1657-1660 